\title{
Identité stricte ou partielle et identification dans les phrases à copule. Comment les identifier?*
}

\author{
Valérie Amary-Coudreau \\ Normandie Université, Unicaen, CRISCO (EA4255) \\ valerie.amary@unicaen.fr
}

\section{Introduction}

Le terme d'identité recouvre une large palette d'interprétations en fonction des domaines dans lesquels on l'envisage. Dans le langage courant, on parle d'identité d'une personne, ce qui recouvre sa date et son lieu de naissance, sa nationalité, son sexe (ou genre), sa situation professionnelle et/ou sociale, etc. Or, un individu ne cesse d'avoir la même identité tout au long de sa vie, alors que la plupart de ses cellules ne sont plus mêmes, et alors que l'individu a pu se transformer mentalement de sorte qu'il ne soit plus réellement le même! Pourtant, une personne garde son identité toute sa vie, sauf accident de parcours, une identité qui, aussi, se construit au fur et à mesure de la vie. Beaucoup d'études ont été proposées sur les référents évolutifs, animés ou non (cf. Charolles \& Schnedecker, 1993, Reboul, 1997). En mathématiques, une identité est une égalité vraie quelles que soient les valeurs données aux inconnues de part et d'autre du signe « $=»$. En logique et en philosophie, l'identité correspond au fait que deux choses/personnes/notions ne font qu'une ou qu'une chose/personne/notion est identique à elle-même (cf. Frege, 1994). La question que nous posons est la suivante : peut-on parler d'une identité en langue ? Et si tel est le cas, de quoi parle-t-on?

Selon la taxinomie de Higgins (1973), Les phrases (1) sont généralement considérées comme des identités en anglais et en français. Or, la phrase française pose, selon nous, un problème de validité qui n'a pas fait l'objet de beaucoup d'attention:

(1) a. Cicero is Tully.

b. *Cicéron est Tullius.

En français, il est nécessaire d'ajouter le pronom démonstratif c' dans ce contexte pour rendre la phrase valide :

(2) Cicéron, c'est Tullius.

Or, souvent la phrase (1)b est donnée comme correcte ${ }^{1}$, ce que l'on peut sans doute en partie expliquer par l'influence de l'anglais, même si d'autres hypothèses doivent être envisagées (cf. Amary-Coudreau, 2010, 2011). On peut noter que la négation rend la phrase en est possible (Cicéron n'est pas Tullius), mais alors on n'a plus d'identité. Par ailleurs, on trouve des phrases classées comme des identités alors qu'elles ne semblent pas correspondre à cette sous-classe de phrases copulatives (cf. Higgins, 1973) :

(3) a. The number of planets is nine.

b. Socrate is a philosopher.

On se trouve donc à la fois devant un problème de forme concernant le français (présence quasiobligatoire du démonstratif, selon nous) et devant un vide concernant la définition de l'identité en langue de façon générale. Notre analyse, qui se situe à l'interface syntaxe-sémantique, tente d'appréhender les caractéristiques formelles des différentes interprétations que l'on peut trouver avec le verbe être afin de délimiter le champ particulier de l'identité et d'en proposer une description.

Notre hypothèse est la suivante : l'identité se présente sous la forme d'une phrase en c'est où les deux termes possèdent exactement la même structure interne, et par conséquent le même degré de référentialité. Dans la section 2, nous présentons le cadre théorique, notamment la classification de 
Higgins (1973) ainsi que les hypothèses envisagées concernant la présence du démonstratif sur la base de Heycock \& Kroch (1999). La section 3 montre que la définition mathématique de l'identité ne permet pas de distinguer l'identité des autres sous-catégories de phrases. La section 4 fait une petite mise au point sur les phrases données comme des identités et traitées par les logiciens et philosophes en termes de vrai ou faux. Dans la section 5, on donne le cadre général de l'analyse formelle de l'identité, et on se propose de donner des arguments pour la distinguer de la spécification et de l'identification, sur la base de la référentialité mais aussi de la prédicativité. Une fois, la définition de l'identité en langue posée, dans la section 6 , on élabore des tests pour distinguer l'identité stricte et l'identité partielle, et les distinguer aussi de l'identification.

\section{Cadre théorique et hypothèses envisagées}

\subsection{Taxinomie de Higgins (1973)}

Notre étude s'appuie sur les données françaises, en prenant en compte la taxinomie des phrases copulatives de Higgins (1973), laquelle distingue quatre sous-catégories de phrases copulatives:

(4) a. prédication: John is happy/a linguist.

b. spécification: The best cook is John.

c. identification: This man is John.

d. identité: Cicero is Tully.

Depuis Higgins (1973), beaucoup de travaux ont porté sur ce classement, soit en essayant de réduire le nombre de sous-catégories à trois (cf. Mikkelsen, 2004) ou en encore à deux, prédicationnelles et équatives (cf. Heycock \& Kroch, 1999), tandis que Declerck (1988) a proposé d'ajouter une souscatégorie $^{2}$. En français, la spécification, l'identification et l'identité font apparaître le pronom démonstratif neutre $c^{\prime}$. Le verbe est tout seul n'est possible que pour la prédication:

(5) a. prédication: Jean est heureux/linguiste.

b. spécification : Le meilleur cuisinier, *(c')est Jean.

c. identification : Cet homme, *(c')est Jean.

d. identité : Cicéron, *(c')est Tullius.

Certains auteurs (cf. Mikkelsen, 2004, parmi d'autres) notent un manque de définitions pour ces souscatégories de phrases copulatives, néanmoins on peut proposer les descriptions suivantes :

Les phrases prédicationnelles sont les phrases où le terme post-copule (XP2) est un prédicat et où le prédicat dit quelque chose à propos du sujet $(\mathrm{XP} 1)^{3}$.

Les phrases spécificationnelles sont souvent définies comme des phrases où le terme post-copule XP2 est référentiel, tandis que le constituant pré-copule XP1 est dit prédicatif. De ce fait, certaines études analysent les spécificationnelles comme une inversion des phrases prédicatives (cf. Moro, 1997). Mais cette analyse est remise en cause par certains auteurs, notamment Heycock \& Koch (1999) selon qui les deux XP de la spécification sont référentiels. Pour Higgins (1973), le XP2 vient spécifier la référence d'une variable laissée ouverte par le XP1.

Les phrases identificationnelles, proches des spécificationnelles, présentent un terme post-copule référentiel qui identifie la référence du XP1, cette référence étant posée comme unique contrairement aux phrases spécificationnelles. Mais le XP1 est considéré comme référentiel dans les identificationnelles, alors qu'il est posé comme prédicatif pour les spécificationnelles.

Les phrases à identité présentent deux termes référentiels mis en relation, dont on pose l'équivalence. Higgins (1973) utilise, quant à lui, l'appellation « identité » alors que le mot « équatif » pour ces phrases est largement utilisé dans la littérature générative (cf. Mikkelsen, 2004, den Dikken, 2006).

Comme on peut le voir, les trois sous-catégories spécification, identification et identité peuvent être envisagées comme contenant deux termes référentiels (sauf pour Higgins, 1973, mais nous laisserons sa 
terminologie, assez particulière, de côté ${ }^{4}$ ). Notre objectif est de montrer que, malgré une similitude forme (Topique c'est Focus), les identités se distinguent de la spécification et de l'identification.

\title{
2.2 La présence quasi-obligatoire du démonstratif $c^{\prime}$ dans les équatives en français?
}

Certains locuteurs (et certains linguistes) ont tendance à accepter les phrases (5)b-d sans le pronom démonstratif, cependant ces jugements posent problèmes, les phrases avec est tout seul apparaissent peu naturelles voire artificielles (cf. Le Goffic, 1993 : 208-210). Culioli \& Desclès (1981) font le même type de remarque :

\begin{abstract}
«Remarquons que l'on a assez difficilement en français, sauf pour les logiciens qui construisent des métatextes ou pour des mauvais observateurs : ? Vénus est l'étoile du matin (plutôt : Vénus, c'est l'étoile du matin). » (cf. Culioli \& Desclès, 1981 : 90-91)
\end{abstract}

Et si Apothéloz (2008) donne un exemple sans le démonstratif, ce n'est que pour mieux constater qu'il n'est pas très naturel et pourrait constituer une sorte d'hypercorrection :
«(10) Ce qui a ajouté au désarroi de la population est l'adoption, et son entrée immédiate en vigueur, d'une loi sur un impôt supplémentaire destiné à réunir des fonds pour financer l'engagement de l'armée au Kosowo. (Libération, 8.10.1998) Valli (1981) a discuté d'exemples comme (10) et formulé l'hypothèse que cette forme de la copule équative pourrait, du moins dans certains cas, être un indice sociolinguistique d'hypercorrection. » (cf. Apothéloz, $2008: 80$ )

En effet, il n'existe aucune « règle » explicite interdisant le fait d'employer est tout seul dans les phrases non-prédicationnelles, néanmoins elles apparaissent beaucoup plus spontanées avec le démonstratif $c$ ' à l'oral ainsi qu'à l'écrit. Le fait qu'en anglais is (seul) soit employé dans toutes ces structures peut aussi influencer les jugements sur le français. Il est aussi possible que des critères intonatifs (accentuation, pause), qui peuvent rendre ces phrases acceptables à l'oral en leur donnant des interprétations diverses, puissent être intégrés par le locuteur lecteur pour qu'il soit susceptible de les accepter en les lisant.

\subsection{Is vs. c'est dans les équatives, pourquoi ?}

Pourquoi trouve-t-on is en anglais dans les phrases copulatives non prédicationnelles alors qu'en français, il faut ajouter un démonstratif à est pour que ces phrases soient naturelles ? On peut trouver un argument dans l'hypothèse de Heycock \& Kroch (1999) qui montrent que les phrases prédicationnelles et les trois autres sous-catégories de phrases présentent des structures de surface différentes même si en anglais cette distinction n'est pas visible. Ces auteurs dissocient donc deux sous-catégories dans le classement de Higgins (1973): d'un côté les phrases prédicationnelles et d'un autre côté les spécifications, identifications et identités, regroupées sous l'appellation d'équatives. Les équatives présentent une structure Topique is Focus, alors que pour les prédicationnelles le XP1 est dans la position canonique du sujet. On peut représenter cela de la façon suivante sous une forme simplifiée :

(6) a. Les prédicationnelles :

b. les équatives (ici une identité) :
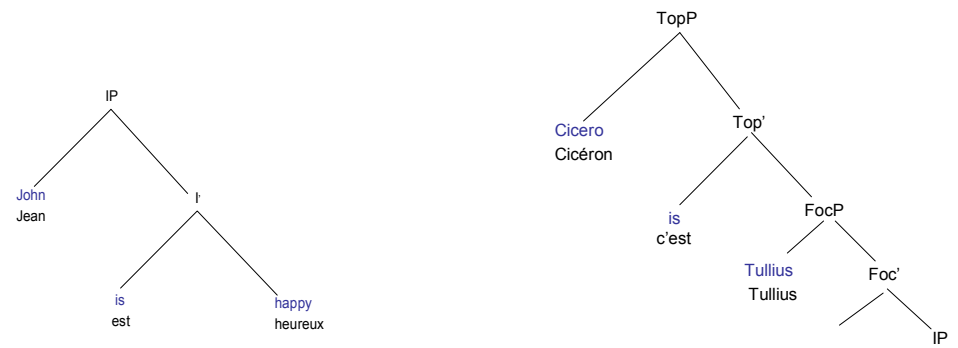
Même s'il existe d'autres facteurs intervenant dans cette problématique, il apparait qu'en anglais is puisse se déplacer dans la position tête de la projection Topique, et jouer le rôle d'un marqueur de topique (cf. Lee, 2002), alors qu'en français est n'a pas cette possibilité et qu'il doit être associé au démonstratif $c$ ' pour pouvoir remplir ce rôle. Il semble qu'il y ait une répartition des tâches entre le démonstratif et le verbe être. Le verbe être permet notamment de marquer le temps, et le démonstratif $c$ ' en se cliticisant sur est permet d'activer le déplacement vers la tête Topique ainsi que la mise en relation de deux termes référentiels via la structure Topique c'est Focus. Nous avons avancé l'hypothèse (cf. Amary-Coudreau, 2010) que les équatives, regroupant la spécification, l'identité et l'identification, ne sont pas des cas de phrases disloquées, et que le démonstratif n'y constitue pas un pronom référentiel résomptif mais y joue le rôle du marqueur de Topique, comme c'est le cas pour to en polonais ou éto en russe (cf. Citko, 2008, Markman, 2008).

Selon Heycock \& Kroch (1999), la spécification, l'identification et l'identité n'ont pas à être distinguées parce qu'elles mettent toutes en jeu des syntagmes pré- et post-copules qui sont référentiels. Bien que nous adoptions le schéma général proposé par Heycock \& Kroch (1999), nous ne sommes pas d'accord sur le fait que les XP1 et XP2 aient le même statut référentiel dans les trois sous-catégories d'équatives. Nous proposons que les identités soient à part, dans le sens où elles font intervenir une contrainte particulière: les deux XP, dans $X P 1$ c'est $X P 2$, doivent être exactement de même nature syntaxique (linéairement parlant). Les notions de référentialité et de prédicativité, prépondérantes pour la spécification, l'identification et la prédication, ne jouent, selon nous, pas le même rôle pour l'identité.

\section{Spécification, identification, identité et les mathématiques}

Nous avons proposé que les équatives se distinguent des phrases prédicationnelles en français par la présence quasi-obligatoire du démonstratif $c e$. Selon Higgins (1973), l'identité relie deux XP référentiels, même s'il reste très prudent sur l'existence d'identités en langue(s), ainsi que ce qui peut être considéré comme référentiel. L'identité serait, selon Higgins, la seule sous-catégorie de phrases copulatives à avoir deux XP référentiels. Nous pensons que la notion de référentialité n'est pas en jeu de la même façon pour les phrases à identité que pour les autres sous-catégories, point sur lequel nous revenons par la suite. Mais la notion d'identité semble tellement liée aux mathématiques que nous souhaitons ici tenter d'appliquer les trois caractéristiques de l'identité mathématique aux équatives : à savoir la substitution ou «être la même chose », la symétrie et la transitivité.

Une identité pose que deux individus ou objets ne forment, en réalité, qu'un seul et même individu ou objet, qu'ils sont « la même chose ». Mais alors que signifie " être la même chose » (pour une étude de même, cf. Van Peteghem, 1997)? Les spécificationnelles et les identifications posent en principe également que deux individus/objets sont « la même chose » :

(7) Spécificationnelles : a. The winner is John. $\Rightarrow$ the winner and John are the same person.

a'. Le gagnant, c'est Jean. $\Rightarrow$ le gagnant et Jean, c'est la même personne.

b. What I don't like about John is his tie. $\Rightarrow$ what I don't like about John and his tie are the same thing.

b'. Ce que je n'aime pas chez Jean, c'est sa cravate. $\Rightarrow$ Ce que je n'aime pas chez Jean et sa cravate, c'est la même chose

(8) Identification: This man is John. $\Rightarrow$ This man and John are the same person.

Cet homme, c'est Jean. $\Rightarrow$ Cet homme et Jean, c'est la même personne.

(9) Identité: Cicero is Tullius. $\Rightarrow$ Cicero and Tullius are the same person.

Cicéron, c'est Tullius. $\Rightarrow$ Cicéron et Tullius, c'est la même personne.

Alors, qu'est-ce qui est spécifique aux identités ?

Les identités en mathématiques sont des équations qui sont (posées comme) vraies quel que soit le contexte, où les deux termes mis en relation peuvent être intervertis, pour satisfaire la règle de symétrie. Or, en raison de l'asymétrie du langage, il ne peut exister, en langue, des identités, telles qu'elles sont décrites pour des mathématiques. Si je dis Emile Ajar, c'est Romain Gary, ou Romain Gary, c'est Emile Ajar, je ne dis pas la même chose. La première phrase est au sujet de Emile Ajar, qui en est le topique, la 
deuxième phrase est au sujet de Romain Gary, qui en est le topique. Ainsi les deux phrases ne peuvent pas avoir le même contexte dans une langue naturelle. Emile Ajar et Romain Gary ne peuvent pas être intervertis. Une phrase comme Emile Ajar, c'est Romain Gary ne peut pas avoir la même représentation que la «phrase » mathématique « Emile Ajar = Romain Gary ».

Pour ce qui concerne la transitivité, les spécificationnelles et les identifications répondent à cette exigence aussi bien que les identités :

(10) a. If the winner is my friend and my friend is John, then the winner is John.

a'. Si le gagnant, c'est mon ami et mon ami, c'est Jean alors le gagnant, c'est Jean.

b. If this is this man and this man is John, then This is John.

b'. Si (ça) c'est cet homme et cet homme, c'est Jean, alors (ça) c'est Jean.

c. If Peter is John and John is Bill, then Peter is Bill.

c'. Si Pierre, c'est Jean et Jean, c'est Paul, alors Pierre, c'est Paul.

Ainsi les mathématiques ne peuvent pas nous aider à décrire ce que pourrait être une identité en langue, alors que nous savons intuitivement que dire The winner is John, that man is John, et Bill is John n'implique pas la même interprétation et que ces phrases ne pourraient pas être énoncées dans le(s) même(s) genre(s) de contextes. Il semble que l'identité fasse une certaine sorte de comparaison et pose que deux dénotations avec deux références ne font qu'une seule et même chose. La spécificationnelle a un XP1 avec une propriété comme référence qui a besoin d'une dénotation, alors que l'identification a une référence qui a besoin d'une dénotation.

Ainsi si nous sommes sur la bonne voie, l'identité serait la seule sous-catégorie de phrases copulatives à être une comparaison. Or, seules des choses comparables peuvent être comparées. Nous souhaitons montrer que la comparaison a lieu entre deux éléments ayant exactement la même structure syntaxique interne. Ni les types sémantiques généraux $<\mathrm{e}>$ ou $<\mathrm{e}, \mathrm{t}>$ (cf. Partee, 1987), ni les catégories syntaxiques générales (DP, CP) ne sont suffisantes. La description doit être plus précise pour expliquer les faits.

\section{Jugement d'identité, logique, vérité et petits mensonges}

Qu'appelle-t-on identité lorsque l'on parle de phrases avec le verbe être? Il semble que tout le monde ne s'accorde pas sur ce point. Un exemple flagrant est discuté par Higgins (1973) :

(11) The number of planets is nine.

Selon Higgins (1973 : 199-203), la phrase (11) ne constitue pas une identité, mais une spécification parce que le premier terme the number of planets n'y est pas référentiel. Un autre exemple de "fausse identité" souvent traité par les logiciens (dont Russel) est dénoncé par Higgins (1973: 269) :

(12) Socrate is a philosopher.

Ici, a philosopher représente un prédicat pour Socrate et cette phrase est prédicationnelle. Il ne peut donc pas s'agir d'une identité.

Pour ce qui est du traitement logique, Higgins (1973) avait déjà noté qu'il s'agissait d'une impasse si l'on se place du point de vue de la langue et avait dénoncé le fait que les phrases employées par les logiciens comme des identités ne sont, en fait, pas des identités. Donc, on a ici deux types de problématiques: le traitement logique de phrases en langues naturelles, et la description (faute d'une "définition") de ce que pourrait être une identité en langue. Il est, à ce propos, tout à fait amusant que les recherches en astronomie aient démontré, il y a quelques années, que l'un des plus célèbres exemples donnés par les logiciens (11) est faux ${ }^{5}$. Or, l'objet des logiciens et des philosophes n'est pas la langue en elle-même, et ce qui compte comme étant une identité pour eux n'est pas une identité en langue naturelle (si jamais une telle chose existe). On pourrait considérer que quelque chose comme (13) soit une identité, mais (13) n'est pas une phrase appartenant à une langue naturelle même s'il est possible de l'apparenter à une "phrase" mathématique:

(13) the number of planets $=$ nine 
Il est intéressant de noter que la traduction de "=" par "est" en français est difficile:

(14) ???Le nombre de planètes est neuf. ${ }^{6}$

Or, en logique, les "identités" sont la plupart du temps traitées en termes de "vrai" ou "faux". Pourquoi les chercheurs qui travaillent sur la langue présument-ils que lorsque nous parlons, nous disons toujours la vérité? Il semble, au contraire, que l'une des habiletés des êtres humains ${ }^{7}$, doués de langage, soit de ne pas dire la vérité. Et il apparaît aussi que le langage humain nous donne cette "compétence" du mensonge ${ }^{8}$ ou de la non-vérité. Ce n'est pas parce que la notion d'identité s'appuie (à tort sans doute, comme on vient de le voir) sur des bases mathématiques qu'il faudrait croire que les identités en langue se comportent de la même manière. Quand bien même ce serait le cas, les mathématiques et la logique doivent déterminer ce qui est vrai et ce qui est faux. Mais est-ce l'objectif des sciences du langage ? La tâche du linguiste consiste à répondre aux questions suivantes (parmi d'autres): est-ce que cette phrase peut-être ou non produite par un locuteur natif? Qu'est-ce que cette phrase veut vraiment dire? Et pourquoi peut-elle ou doit-elle être dite de cette façon et pas d'une autre? Ce type de questionnement est d'ailleurs généralement utilisé par les linguistes, toutes théories confondues. Pourtant dès qu'il s'agit d'identités, la question primordiale concerne le vrai et le faux, et il n'y a plus de jugements d'acceptabilité qui soient discutés, la forme n'est plus pertinente, et l'interprétation se fond dans le "vrai ou faux". On sait qu'il existe une longue tradition philosophique concernant le verbe être (cf. Derrida, 1971), ainsi d'ailleurs que de nombreux travaux en linguistique depuis Benveniste.

L'idée, très intuitive au départ, et que l'on se propose de formaliser, c'est qu'une identité est posée comme vraie par le locuteur, qu'elle soit réellement vraie ou pas, que locuteur sache qu'elle est fausse (alors il s'agit d'un mensonge ou d'une fiction) ou qu'il ne le sache pas (alors il s'agit d'ignorance). La phrase est donnée comme vraie en elle-même et par elle-même. Ceci implique que le langage et ici, la syntaxe, permet de communiquer des informations fausses (ou pas), mais posées comme vraies, que cela soit intentionnel ou pas. Si je dis Parler, c'est se taire, je pose une identité. D'un point de vue logique, on peut dire que si je parle, alors je ne me tais pas. Donc, la phrase est fausse, pourtant elle prend un sens par ellemême et elle veut vraiment dire quelque chose. On peut d'ailleurs constater que Se taire, c'est parler n'a pas la même interprétation.

Ainsi, ce que l'on souhaite montrer, c'est qu'une identité n'a pas à être traitée nécessairement en termes de vrai et de faux, mais en termes de grammaticalité et doit être envisagée en fonction des constituants qui la composent. Qu'est-ce que l'on peut trouver comme $\mathrm{X}$ et $\mathrm{Y}$ dans une identité $X$ c'est $Y$ ? Qu'est-ce qui distingue une identité d'une spécificationnelle et d'une identification?

\section{Que pourrait être une identité en langue?}

Notre proposition est la suivante : dans une phrase $X P 1$ c'est $X P 2$, dès que la structure syntaxique interne des deux XP n'est pas exactement identique, la nature référentielle/prédicative du XP1 prend le dessus et produit une phrase spécificationnelle ou une phrase identificationnelle. A titre d'exemple, si nous considérons le cas du syntagme nominal, l'ajout d'un adjectif dans un syntagme nominal défini le rend plus prédicatif (il en va de même avec un indéfini), et crée un déséquilibre sur le plan de la référentialité et de la prédicativité. En (15)a, les deux syntagmes ont une forme identique (Déterminant défini + Nom), et on obtient une identité. L'ajout de l'adjectif superlatif pire au premier syntagme le rend plus prédicatif et produit une phrase spécificationnelle cf.(15)b, le même ajout au deuxième syntagme rend la phrase identificationnelle cf.(15)c. Les mêmes caractéristiques apparaissent avec un syntagme nominal dont le déterminant est un possessif cf.(16).

(15) a. Le cauchemar, c'est la maladie. (identité)

b. Le pire cauchemar, c'est la maladie. (spécification)

c. Le cauchemar, c'est la pire maladie. (identification)

(16) a. Son cauchemar, c'est son travail. (identité)

b. Son pire cauchemar, c'est son travail. (spécification)

c. Son cauchemar, c'est son pire travail. (identification) 
Ainsi, on peut en déduire que les deux termes d'une identité doivent avoir le même degré de référentialité, quel que soit ce degré (ce que l'on détaillera au paragraphe 5.2). Bien que notre objectif général (et le moyen d'y parvenir) soit différent de ces auteurs, il semble que notre intuition ne soit pas très éloignée de ce que Culioli \& Desclès (1981) expriment à propos de l'identité et de l'identification :

Dire que l'étoile du matin est Vénus «exprime l'identité (ou l'égalité) de deux expressions » nous paraît cependant une analyse beaucoup trop rudimentaire car il s'agit bien ici d'une identification et non d'une identité. (Culioli \& Desclès, 1981 : 90)

Précisons maintenant nos distinctions. Pour qu'il y a ait identité entre a et $\mathrm{b}$ il est nécessaire qu'à toute occurrence de $\mathrm{a}$ on puisse substituer l'occurrence de $\mathrm{b}$ et réciproquement. On a identification lorsque ce à quoi renvoie a (la valeur référentielle de a) est identifié (il s'agit alors d'une opération) par une énonciation, à ce à quoi renvoie b (la valeur référentielle de b). (Culioli \& Desclès, 1981: 100)

L'identification est une opération, ce qui n'est pas le cas de l'identité. L'identité est une relation (cf. également Dufaye, 2003, et Larreya, 2003).

\subsection{Dissociation entre prédicativité et référentialité}

Il existe de nombreuses études tant sur la notion de prédicat que sur celle de référentialité : qu'est-ce qui est référentiel ? Qu'est-ce qui est prédicatif? Quels sont les indices que nous pouvons trouver ? C'est LE nœud du problème, mais on ne prétendra pas ici répondre à cette question cruciale et obsédante pour quiconque travaille sur ce sujet (cf. la plupart des références de notre bibliographie). Tout en prenant en compte le flou qui règne sur ces notions, notre contribution se borne juste à dissocier l'opposition référentiel/prédicatif : en effet, il est généralement tenu pour acquis que lorsqu'un XP est [+ référentiel] alors il est nécessairement [- prédicatif] et vice versa. Or, comme cela est signalé dans bien des travaux (notamment Rapoport, 1987, mais aussi Higgins, 1973), un même XP peut être référentiel et prédicatif. Higgins (1973), quant à lui, reste extrêmement prudent sur ces notions, et montre bien qu'un même SN peut avoir plusieurs types d'interprétation. Il suggère aussi la possibilité d'un continuum qui irait de l'expression référentielle au prédicat (attributif dans ses termes) (cf. Higgins, 1973 : 206). L'idée de ce continuum est aussi présente chez Heggie (1988) qui propose une hiérarchie pour évaluer ce qui peut servir d'attribut à chaque type XP en fonction de leur nature référentielle respective. Or, ce que nous proposons n'est pas un continuum allant de référentiel à prédicatif, mais une dissociation des deux notions. Notre proposition provient de l'observation que deux XP peuvent être classés comme référentiels alors qu'ils ont un comportement différent quant à la prédicativité. Mon ami ou my friend peuvent référer à quelqu'un en particulier, et donc être référentiels, mais ils peuvent aussi constituer des prédicats, et à ce titre correspondre à une caractéristique, à une propriété et non plus à un individu particulier. En français, on peut observer cela de façon directe : mon ami peut être un prédicat dans une phrase prédicationnelle, alors qu'il peut être référentiel dans une phrase identificationnelle :

(17) a. Pierre est mon ami. (prédication)

b. Pierre, c'est mon ami. (identification)

Les deux phrases ne peuvent pas être employées dans les mêmes contextes : la phrase identificationnelle correspond, par exemple, à une réponse à une demande du type "C'est qui Pierre? ». La phrase prédicationnelle semble pouvoir apparaître plutôt dans un contexte de justification...

L’anglais est ambigu et (18) peut correspondre aux deux phrases françaises (17) :

(18) Peter is my friend.

D'un autre côté, un nom propre comme Pierre ne peut pas constituer un prédicat ${ }^{9}$. En français, Pierre ne peut pas apparaître comme le prédicat d'une phrase prédicationnelle :

(19) a. *Mon ami est Pierre.

b. *Il est Pierre. 
Ainsi mon ami peut être à la fois référentiel et prédicatif, et de ce fait, constitue un bon candidat pour la phrase spécificationnelle :

(20) Mon ami, c'est Pierre.

Les phrases spécificationnelles sont quelquefois analysées comme des phrases prédicationnelles inversées (cf. Moro, 1997, Mikkelsen, 2005), ce qui peut être le cas de (18) en anglais mais pas en français cf.(19)b.

(21) My friend is Peter.

L'argument de Heycock \& Kroch (1999) contre cette hypothèse repose sur le fait que si la spécificationnelle était inversée par rapport à la prédicationnelle, la phrase (22)b devrait être bonne, ce qui n'est pas avéré :

(22) a. John is proud of his daughters.

b. *Proud of his daughters is John.

Le topique d'une phrase spécificationnelle doit à la fois être porteur de référentialité et avoir une valeur prédicative. Mais si l'on a que deux choix, soit [+ référentiel, - prédicatif] ou [- référentiel, + prédicatif], il n'est pas possible de rendre compte des données. Ainsi, tout semble bloqué par la dialectique [+ prédicatif] $\Rightarrow$ [- référentiel]. Pourtant, on peut trouver une sortie à cette impasse en proposant que la valeur prédicative d'un XP soit indépendante de sa valeur référentielle.

Observons quelques arguments très simples en faveur de cette proposition. Si nous considérons l'explétif français $i l, i l$ est [-prédicatif], mais il est aussi [- référentiel]. Ainsi, il n'est pas nécessaire d'opposer la référentialité à la prédicativité. Rien n'empêche un élément qui a une valeur prédicative positive d'avoir aussi une valeur référentielle positive. Un XP défini du type $L e N$ peut être référentiel, mais aussi avoir une valeur prédicative: le type de $\mathrm{N}$ intervient ici le problème ou le gagnant aura une valeur plus prédicative que la table ou le linguiste, mais il ne s'agit pas du seul facteur. Les présupposés et/ou les modifieurs possibles à l'intérieur du syntagme nominal permettent aussi de transformer la valeur prédicative du syntagme: le meilleur linguiste du monde sera plus prédicatif que le linguiste.

Pour les valeurs de prédicativité, nous donnons ci-dessous quelques tests permettant un classement entre [-prédicatif], [+ prédicatif] et [+/- prédicatif] pour quelques types de syntagmes nominaux (en laissant de côté d'autres catégories syntaxiques ${ }^{10}$ ). Ainsi, selon Higgins (1973), le verbe devenir ne peut prendre qu'un complément de type prédicatif.

Un XP + prédicatif est un XP qui peut être en position complément de devenir mais qui ne peut pas jamais être dans une position argument.

(23) a. Léon est devenu heureux/musicien.

b. *J'ai vu heureux/musicien.

Un XP - prédicatif est un XP qui ne peut pas être en position complément de devenir mais qui peut être dans une position d'argument.

(24) a. *Il est devenu Léon.

b. *Il est devenu ce musicien. (mais : il est devenu cet excellent musicien que l'on connaît)

c. *Il est devenu le musicien. (mais : il est devenu l'excellent musicien que l'on connaît)

d. *Léon est devenu un musicien. (mais : Léon est devenu un excellent musicien)

(25) J'ai vu Léon/ce musicien/le musicien/un musicien.

Un XP est +/- prédicatif s'il peut être prédicatif dans certains contextes (test avec devenir) et argument dans d'autres (comme complément d'un verbe) ou encore s'il peut être complément d'un verbe comme s'interroger sur (cf. Schlenker, 2003) :

(26) Il est devenu mon meilleur ami/ un excellent musicien/ l'excellent musicien que l'on connaît.

(27) J'ai vu mon meilleur ami/ un excellent musicien/l'excellent musicien que l'on connaît. 
(28) Je m'interroge sur mon meilleur ami (est-ce que j'ai un meilleur ami?)/ ??un musicien/un excellent musicien (qui pourrais-je connaître qui serait un excellent musicien)/??le musicien/ le meilleur musicien (quel est-il ?).

Dans les phrases (28), l'interrogation porte sur le fait que l'on ignore quelque chose à propos du XP, tandis que l'interprétation « $j$ 'ai des doutes sur $X P$ » reste disponible.

On peut ainsi distinguer différentes classes de prédicativité cf.(29), sous forme d'un continuum indépendant du continuum de la référentialité (cf.(30), on s'appuie ici sur Heggie, 1988) ${ }^{11}$, ce que l'on peut mettre sous forme d'ébauche de la façon suivante ${ }^{12}$ :

(29) Prédicativité : déictiques, noms propres, Le N, Ce N, Un N, il (valeur [-])] $<$ Le N + Modifieur, mon $\mathrm{N}$, un $\mathrm{N}+$ Modifieur $($ valeur $[+/-])<\mathrm{N}$, adj $($ valeur $[+])$.

(30) Référentialité : déictiques $>$ noms propres $>$ ce $\mathrm{N}$ (non déictique) $>$ le $\mathrm{N}$, mon $\mathrm{N}>$ un $\mathrm{N}>$ il (non explétif) $>\mathrm{N}$, Adj.

On posera les principes généraux suivants pour les phrases à copule :

(31) Principes généraux :

a. Pour les spécificationnelles : XP1 dans XP1 c'est XP2 doit être +/- prédicatif.

b. Pour les identifications : XP1 dans XP1 c'est XP2 doit être + référentiel.

Il ne s'agit pas ici de détailler ces principes généraux (ce qui est fait par ailleurs), mais de proposer une description précise de chacune des sous-catégories de phrases copulatives afin de trouver un moyen de décrire l'identité par rapport aux autres catégories. Notre hypothèse permet de rendre compte de la nature distincte du premier terme des phrases spécificationnelles, en posant qu'il s'agit d'un terme [+/prédicatif] ce qui bloque les termes [+ prédicatif] comme les adjectifs, et de rendre compte du trait Topique que doit posséder le premier terme par sa valeur référentielle, laquelle n'est pas impliquée par sa valeur prédicative.

La spécification n'est pas le thème de la présente étude, cependant il apparaît nécessaire de distinguer ce type de phrases de l'identification et l'identité (contra Heycock \& Kroch, 1999) pour avancer dans une description de l'identité. On posera les principes suivants :

(32) a. Si X et Y n'ont pas exactement la même structure syntaxique dans $X$ c'est $Y$, interpréter comme une spécification ou une identification en fonction de la nature prédicative et référentielle de $X$.

b. Si $\mathrm{X}$ et $\mathrm{Y}$ ont exactement la même structure syntaxique dans $X$ c'est $Y$, interpréter comme identité.

Naturellement, en proportion, on trouve moins de cas où $\mathrm{X}$ et $\mathrm{Y}$ présentent la même structure interne que de cas où $\mathrm{X}$ et $\mathrm{Y}$ ont des structures différentes. Et sur les plans sémantique et pragmatique, les conditions qui permettent un énoncé de type identité posent des restrictions importantes: il faut que deux personnes/objets/concepts n'en fassent qu'un(e) ou bien soient donnés comme n'en faisant qu'un(e). Cela suppose que le locuteur présuppose que l'interlocuteur ne le sache pas ou se trompe et l'ajout de «en fait » ou «en réalité » produit un énoncé plus naturel dans certains cas. Selon Desclès \& Froidevaux (1982: 80-81), «Les langues naturelles ne marquent pratiquement jamais l'identité, alors qu'il existe plusieurs procédés pour signifier l'identification. ». Nous ne sommes que partiellement d'accord avec ces auteurs : selon la définition que nous en donnons, on trouve des identités en langue que l'on peut nettement distinguer de l'identification.

\subsection{Une structure interne identique pour les deux XP}

Ainsi, selon le principe posé en (32)b, si deux XP ont la même structure syntaxique interne, on obtient une identité, quelle que soit la catégorie du XP:

(33) a. Un médecin, c'est un voleur.

b. Le médecin, c'est le voleur.

c. Mon médecin, c'est mon ami. 


\author{
d. Marcel, c'est M. Noël \\ e. Noir, c'est noir. \\ f. Vivre, c'est souffrir. \\ g. Ce que j'aime, c'est ce que tu fais. \\ h. Voleur, c'est tricheur.
}

Cela va à l'encontre de ce qui est proposé par Higgins (1973: 245), selon qui, dans une identité, les deux membres doivent être référentiels, même s'il reconnaît avoir des doutes à propos de la distinction entre identité et identification. Il est pourtant généralement reconnu que les identités admettent deux XP référentiels (sauf pour Heycock \& Kroch, 1999), sans prendre en compte la structure interne de ces XP.

\title{
5.3 Peu importe la proximité sémantique des deux XP de l'identité
}

Parallèlement à cela, il existe un fait très intrigant noté dans Barbaud (2006). Barbaud (2006) montre à travers les exemples suivants que le fait de poser une identité entre deux termes dont les sens sont extrêmement proches ne rend pas les phrases valides pour autant:

(34) a. *La prédiction, c'est expliquer.

b. *?La mort, c'est (finir, ?disparaître)

c. *La peinture, c'est colorier.

d. ?Le braconnage, c'est voler.

On pourrait pourtant considérer que la proximité sémantique des infinitifs et des syntagmes nominaux devrait permettre une identité ou même une identification. Or ces phrases sont tout simplement agrammaticales. On peut transformer les exemples (34) de Barbaud (2006) en mettant le nom et le verbe correspondant, les phrases obtenues restent non valides :

(35) a. *La prédication, c'est prédiquer.

b. *La mort, c'est mourir.

c. *La peinture, c'est peindre.

d. *Le braconnage, c'est braconner.

Même si les phrases (35) comme celles de (34) peuvent recevoir une interprétation, il n'en reste pas moins vrai que ce sont des phrases mal formées. Ainsi, le sens rationnel apparaît comme non suffisant pour construire une identité (et sans doute non nécessaire), et la forme semble être prépondérante. Or, les phrases (34) deviennent valides si le premier XP est aussi un infinitif :

(36) a. Prédiquer, c'est expliquer.

b. Mourir, c'est (finir, disparaitre).

c. Peindre, c'est colorier.

d. Braconner, c'est voler.

Ici, dans les exemples de Barbaud (2006), les deux infinitifs ont une relation très proche au niveau de leur sens, mais on peut très bien imaginer de relier deux verbes dont les significations n'ont rien en commun. Le simple fait de les unir avec c'est permet de constituer une identité valide sur le plan syntaxique et interprétable :

(37) a. Parler, c'est se taire.

b. Manger, c'est pédaler.

c. Ignorer, c'est crier.

d. Défaire, c'est tousser.

Que l'identité soit absurde ou pas au niveau sémantique ne change rien au fait qu'elle soit posée comme vraie. Les exemples précédents contiennent seulement des infinitifs, mais le même phénomène peut être observé quelle que soit la nature des constituants :

(38) a. Vert, c'est rouge.

b. La mort, c'est le fauteuil.

c. Ce que j'aime, c'est ce que je déteste.

d. Mon rideau, c'est ma robe. 
e. Léon, c'est Gertrude.

Même si ces phrases ainsi construites peuvent sembler absurdes, on peut leur attribuer une interprétation, et si un locuteur les énonce, il pose qu'elles sont vraies pour lui. A l'opposé, on peut dire quelque chose qui n'est pas absurde au niveau sémantique, mais si les deux XP n'ont pas la même structure interne, on n'obtient pas une identité:

(39) a. Mon médecin, c'est une personne qui me soigne.

b. Léon, c'est l'homme que j'aime.

(39)a est interprété soit comme une spécification soit comme une identification, et (39)b est interprété comme une identification, en raison de la nature du XP1 qui peut être à la fois [+ référentiel] et [+/prédicatif] en (39)a et seulement [- prédicatif] in (39)b.

Notre définition de l'identité en langue est donc une définition formelle : dès que l'on a une phrase avec deux constituants de même nature reliés par c'est, on l'interprète comme une identité.

\section{L'interprétation : identité stricte et identité partielle}

Néanmoins quant à l'interprétation des identités telles nous venons de les définir de façon formelle, il existe certaines disparités, que nous nous proposons d'étudier. Larreya (2003) distingue des identifications strictes et partielles, et selon lui, cette distinction ne peut pas s'appliquer à l'identité. Dans ce qui suit, nous proposons des tests montrant que l'identité peut être stricte ou partielle, alors que ce n'est pas le cas de l'identification.

\subsection{Test : XP1 c'est XP3 et/mais c'est aussi XP2}

Observons les exemples suivants :

(40) a. La guerre, c'est la guerre.

b. Marcel, c'est M. Noël.

c. Vivre, c'est souffrir.

Si l'on effectue une transformation de ces phrases sur la base de $X P_{1}$ c'est $X P_{3}$,et/ mais c'est aussi $X P_{2}$, on obtient une phrase non pertinente pour (40)b et des phrases valides pour (40)a et c :

(41) a. La guerre, c'est une lutte pour la paix, mais c'est aussi la guerre.

b. Marcel, c'est un homme, \# mais c'est aussi M. Noël.

c. Vivre, c'est respirer, manger, mais c'est aussi souffrir.

Si l'on interprète ces phrases ainsi formées (pour celles qui sont valides), cela signifie que XP1 c'est aussi autre chose que XP2, ce que l'on peut encore paraphraser par: mais ce n'est pas que ça, cf. les phrases transformées en (42).

(42) a. La guerre, c'est la guerre, mais ça n'est pas que ça.

b. Marcel, c'est M. Noël, \# mais ça/il n'est pas que ça.

c. Vivre, c'est souffrir, mais ça n'est pas que ça.

Là encore (42)b s'oppose à (42)a et c. Si une chose n'est pas autre chose que ce qu'elle est, on peut considérer que l'on a affaire à une identité stricte, c'est le cas de la phrase (40)b, mais ce n'est pas le cas pour les phrases (40)a et c qui expriment que XP1 c'est XP2 mais pas seulement. On pourrait alors définir les identités (40)a et c comme des identités partielles. Le cas de la tautologie comme identité partielle peut apparaître comme un résultat étrange, nous lui consacrons un paragraphe à part.

Le fait qu'une identité soit stricte n'est pas dépendant du fait que la référence soit [-humain] ou [+humain]. Sachant que loup et bar sont deux noms différents attribués au même poisson, on obtient les phrases suivantes, qui sont des identités strictes ${ }^{13}$ :

(43) a. Un loup c'est un bar.

b. Le loup c'est le bar. 
De même, on peut aussi trouver des identités partielles dont la référence est [-humaine] comme en (40)c mais aussi des identités partielles dont la référence est [+humaine], comme c'est le cas pour la phrase (44)a, à laquelle nous appliquons les transformations précédentes :

(44) a. Mon mari, c'est mon ami.

b. Mon mari, c'est mon compagnon, mais c'est aussi mon ami.

c. Mon mari, c'est mon ami, mais ça n'est pas que ça.

De la façon dont nous avons défini l'identification, on ne peut pas retrouver cette distinction entre stricte et partielle pour cette catégorie de phrases. On ne trouve que l'identification partielle, comme le montre la phrase suivante, ainsi que toutes les autres phrases identificationnelles :

(45) a. François Hollande, c'est le président français.

b. François Hollande, c'est un homme, et/mais c'est aussi le président français.

c. François Hollande, c'est le président français, mais ça/il n'est pas que ça.

Ceci va totalement à l'encontre de ce que propose Larreya (2003), selon qui on peut opposer des identifications strictes à des identifications partielles, alors que la même distinction serait, selon lui, difficile à définir pour l'identité. Cela vient de sa définition de l'identification qui est " l'expression d'une relation d'identité entre deux termes » (cf. Larreya, 2003), ce qui n'est pas ce que nous entendons par identification. Le terme d'identité est défini par Larreya (2003) comme « la relation entre deux termes qui ont même référent », ce avec quoi nous sommes d'accord. Mais, selon nous, le rôle de l'identification n'est pas de poser une identité (l'identité se pose d'elle-même), mais bien de poser une opération mentale qui puisse associer un XP1 (qui a une référence R1) à un XP2 (qui a une référence R2), en disant que la désignation XP2 peut convenir à la référence $\mathrm{R} 1$.

A un niveau superficiel, cela correspond au fait que XP1 a la même référence que XP2, et on aboutit à une identité. Mais l'identité ainsi posée n'est qu'une conséquence de l'identification, elle n'est pas incluse dans l'identification. Pour l'identification, on a deux références avec une coïncidence de ces références. Or, la forme d'un XP participe à la construction de sa référence, et si l'on a deux XP de formes différentes, leur référence n'est pas construite de la même façon. Ainsi même si ces deux références ont en quelque sorte un point d'intersection dans le cadre de l'identification, elles ne sont pas pour autant réduites à ce seul point d'intersection.

En posant que, dans une identité (comme type de phrases copulatives), les deux termes sont de même nature, on élimine les différences de référentiation liées à la nature même des termes.

Dufaye (2003) expose le problème de la manière suivante :

«Pour formuler le problème différemment, on peut se poser la question suivante : comment se fait-il que l'on considère une phrase telle que London is the Capital of England comme une « vraie identification » ou « identification stricte » alors qu'une phrase telle que John is a student est intuitivement ressentie comme une « pseudoidentification » ou « identification partielle »? Sur quoi se fonde l'intuition ?

On peut estimer que l'on se fonde, implicitement ou explicitement, sur un test de symétrisation :

London is the Capital of England. The Capital of England is London.

John is a student. *A student is John.

Toutefois, cette explication n'est pas entièrement satisfaisante dans la mesure où on peut se demander par ailleurs ce qui rend le test de symétrie intuitivement recevable. D'ailleurs, cette symétrie n'est pas totale. Comme on vient de le rappeler, London is the Capital of England et The Capital of England is London sont pareillement acceptables d'un point de vue grammatical. Mais ces deux syntagmes apparaîtront dans des contextes de thématisation différents.»

Pour nous, il n'y a pas d'identification stricte opposée à une identification partielle. Dès lors que l'on a identification, elle ne peut être que partielle. Si nous reprenons l'exemple de Dufaye (2003) en français et que lui appliquons les tests que nous avons précédemment utilisés, il correspond à une identification partielle : 
(46) a. Londres, c'est la capitale de l'Angleterre.

b. Londres, c'est une grande ville mais c'est aussi la capitale de l'Angleterre.

c. Londres c'est la capitale de l'Angleterre, mais c'est aussi la capitale de l'ensemble du Royaume-Uni.

Nous donnons ci-dessous un exemple comparable qui présente une identité stricte :

(47) Leningrad, c'est Saint-Petersbourg .

Selon nous, l'identification consiste en une opération qui permet de donner une désignation à une entité référentielle, dont la référence est posée (ou présupposée ou prétendue) comme non connue. En revanche, l'identité n'a pas à être calculée, elle s'exprime par elle-même, elle pose que deux entités dont la référence est connue ne font qu'une. Ainsi, dès lors que l'on a une identification, la référence des deux termes ne peut être strictement la même et il ne peut y avoir qu'une identification partielle alors que, pour les identités, on peut distinguer entre identité stricte et partielle.

\subsection{Test : En 2014, XP1 c'est XP2}

Pour l'identification (45)a, on peut dire que François Hollande et le Président français correspondent aujourd'hui à la même personne, mais ce n'est pas une identité stricte, puisqu'en 2010 François Hollande n'était pas président, ce que l'on peut montrer par l'ajout d'un complément de temps du type en 2014 :

(48) En 2014, François Hollande, c'est le Président français.

A l'inverse l'identité stricte rend l'ajout d'un complément du type en 2014 totalement saugrenu :

(49) \#En 2014, Marcel, c'est M. Noël.

Pour l'identité partielle, l'ajout de en 2014 semble pertinent et de façon surprenante, c'est aussi le cas pour la tautologie :

(50) a. En 2014, vivre c'est souffrir.

b. En 2014, la guerre c'est la guerre.

\subsection{Test : clivage en c'est...qui}

Par ailleurs, le test du clivage du sujet par l'encadrement en c'est...qui semble peu pertinent pour l'identité stricte, dans la mesure où les deux constituants peuvent également entrer dans ce type de construction, sans pour autant que cela produise des phrases satisfaisantes. Cependant il nous semble que le jugement est identique pour chacune des deux phrases suivantes correspondant à des clivées des phrases (40)b et (43) :

(51) a. ?\#C'est Marcel qui est M. Noël.

b. ?\#C'est M. Noël qui est Marcel.

(52) a. ?\#C'est le loup qui est le bar.

b. ?\#C'est le bar qui est le loup.

L'application de ce test pour l'identification permet en revanche de discriminer le sujet de l'attribut. Pour la phrase (45)a on obtient une clivée en c'est... qui bien formée avec François Hollande en position de focus, et une autre clivée en c'est...qui mal formée avec le Président français en position de focus :

(53) a. C'est François Hollande qui est le Président français.

b. *C'est le Président français qui est François Hollande.

Pour les phrases à identité dont l'identité n'est pas stricte, le clivage produit les phrases suivantes :

(54) a. ???C'est la guerre qui est la guerre.

b. ???C'est vivre qui est souffrir.

c. ???C'est souffrir qui est vivre. 
Comme on peut le voir, l'identité partielle ne réagit pas de la même façon que l'identification au test du clivage : pour ce qui est de la tautologie, on ne peut obtenir qu'une seule phrase, pour laquelle nous ne savons que dire, les trois points d'interrogation marquent surtout le fait qu'elle nous paraît ininterprétable. Pour les phrases (54)b et c, leur validité semble douteuse, ce qui indiquerait qu'on ne peut pas discriminer un « sujet » pour la phrase Vivre c'est souffrir, néanmoins si on forme deux clivées en c'est ... que, l'une est plus adaptée que l'autre :

(55) a. \# ?C'est vivre qu'est souffrir.

b. ?C'est souffrir qu'est vivre.

Ici, la clivée la plus appropriée à la phrase d'origine cf.(55)b ne nous semble pas pouvoir discrimer un « attribut » par rapport à un « sujet » (sinon sa contrepartie en c'est... qui serait valide). En revanche, le test du clivage nous montre que les identités partielles divergent des identifications, et que les deux termes de l'identité partielle sont «plus équivalents» que les deux termes de l'identification qui, clairement, pose les deux termes à deux niveaux différents. Les résultats de ce test montrent aussi qu'il y a sur ce point un fonctionnement similaire entre identité partielle et identité stricte.

\subsection{Les tautologies}

Les tautologies ont fait l'objet de nombreuses recherches (cf. Cadiot \& Nemo, 1997, Riegel, 2001, Gaudin-Bordes, 2008, Bondarenco, 2012, parmi beaucoup d'autres) qui analysent différents types de tautologies sans réelle distinction de forme. On trouve des phrases comme Un sou est un sou, une femme est une femme, ou Paul, c'est Paul analysées peu ou prou sur le même plan. Or, nous avons classé les tautologies en c'est comme des identités partielles, ce qui peut sembler assez surprenant voire contrenature. En effet, dire qu'une chose est elle-même devrait aboutir à une identité stricte. Dans le langage mathématique, cela se traduit par a $=\mathrm{a}$ qui est donné comme étant non-informatif. En disant La guerre c'est la guerre, on n'est pas non-informatif. Tout se passe comme si on segmentait les propriétés liées au référent guerre, et que le premier XP guerre arrivait muni d'une partie de ses propriétés et que le deuxième XP guerre arrivait avec une autre partie de ses propriétés. Ainsi, dire La guerre, c'est la guerre, ce n'est pas ne rien dire. On pourrait paraphraser cette tautologie de différentes façons :

(56) La guerre c'est d'un côté des combats entre des pays, c'est d'un autre côté des gens qui meurent ; La guerre, c'est des gens qui sont victimes, mais c'est aussi des gens qui trouvent le moyen de s'en sortir ; la guerre, c'est des gens qui meurent, mais c'est aussi des gens qui souffrent; mais tout ça c'est encore la guerre.

On pourrait multiplier ce type de paraphrases. Si pour chaque occurrence de la guerre on entend quelque chose de différent, alors les deux occurrences de guerre n'ont pas le même référent. Or, il ne s'agit pas ici de la référence de guerre, mais de plusieurs manières possibles d'entendre ce mot, de plusieurs représentations (au sens de Frege ${ }^{14}$ ) que l'on peut en avoir. Ainsi, en posant dans une structure à identité deux fois le même terme, au lieu d'obtenir une identité stricte, ce que l'on obtient c'est la mise en correspondance de deux sous-ensembles de représentations de ce terme (et/ou de son référent ?), donc une identité qui ne peut être que partielle. Comme on l'a vu, on peut paraphraser cette phrase par La guerre, c'est la guerre, mais ça n'est pas que ça alors que Une femme est une femme peut être paraphrasé par Une femme n'est pas autre chose qu'une femme. Peut-être faudrait-il envisager la tautologie de façon différente pour les phrases en c'est...

\subsection{Récapitulatif des tests}

Récapitulons les résultats de ces divers tests :

Lorsqu'il y a identité stricte :

on ne peut pas transformer la phrase en XP1, c'est XP3 et/mais c'est aussi XP2 ni ajouter : mais ça n'est pas que ça.

l'ajout d'une date (en 2014) produit une phrase saugrenue. 
le clivage en c'est...qui ne produit aucune phrase valide que soit avec l'un ou l'autre des deux constituants.

- il n’y a ni « sujet» ni « attribut».

Lorsqu'il y a identité partielle:

- on peut transformer la phrase en XP1, c'est XP3 et/mais c'est aussi XP2 et on peut ajouter : mais ça n'est pas que ça.

l'ajout d'une date (en 2014) produit une phrase parfaitement interprétable.

- le clivage en c'est...qui ne produit aucune phrase valide que soit avec l'un ou l'autre des deux constituants (comme pour l'identité stricte).

- il n’y a ni « sujet » ni « attribut» (comme pour l'identité stricte).

Pour l'identification (nécessairement partielle):

- on peut transformer la phrase en XP1, c'est XP3 et/mais c'est aussi XP2 et on peut ajouter : mais ça n'est pas que ça (comme pour l'identité partielle).

- l'ajout d'une date (en 2014) produit une phrase parfaitement interprétable (comme pour l'identité partielle).

le clivage en c'est...qui ne produit une phrase valide que pour l'un des deux constituants (contrairement à l'identité partielle).

- l'un des deux XP est « sujet », l'autre est « attribut » (contrairement à l'identité partielle).

\section{En guise de conclusion}

L'identité, en tant que phrase copulative, pose une double problématique : un problème général de forme, et plus particulièrement concernant le français (présence quasi-obligatoire du démonstratif, selon nous) et un vide concernant la définition de l'identité en langue de façon générale.

Une partie de notre analyse porte sur la comparaison entre l'identité en langue et l'identité telle qu'elle est traitée en mathématiques, en logique et en philosophie. Force est de constater que l'identité mathématique constitue un autre objet que l'identité en langue naturelle (notamment parce qu'il n'y a pas de symétrie en langue). Par ailleurs, le traitement logique de l'identité en langue ne s'appuie que sur les valeurs de vérité : d'un côté, la plupart du temps, ce qui est traité comme identité n'en est pas une (cf. The number of planets is nine qui est en fait une spécification selon Higgins (1973)) et, d'un autre côté, ces analyses ne prennent pas en compte le fait que le langage permet de former des phrases qui peuvent apparaître comme fausses du point de vue logique, alors qu'elles sont tout à fait interprétables. C'est le cas notamment d'une phrase comme Se taire, c'est parler. Et, pour ce qui concerne le français, le traitement logique s'appuie sur des données qui ne paraissent pas acceptables comme * Cicéron est Tullius.

Notre analyse de l'identité s'appuie sur la taxinomie de Higgins (1973). Nous proposons une description des différents types de phrases copulatives en français, qui fait apparaître une dichotomie entre phrases prédicationnelles d'un côté (phrase en est) et phrases équatives de l'autre (phrases en c'est), suivant l'hypothèse de Heycock \& Kroch (1999) concernant la différence de structure entre ces deux types de phrases : Sujet is/est Prédicat pour les prédicationnelles et Topique is/c'est Focus pour les équatives.

Néanmoins, contra Heycock \& Kroch (1999), nous proposons que l'identité se distingue des deux autres sous-classes d'équatives (spécifications et identifications). Selon nous, l'identité se présente sous la forme d'une phrase en c'est où les deux termes possèdent exactement la même structure interne, et par conséquent le même degré de référentialité. Ceci nous permet de distinguer l'identité de la spécification et de l'identification, pour lesquelles X et Y ont des formes différentes. Dans ces derniers cas, les valeurs de 
prédicativité et de référentialité de X permettent de classer la phrase soit comme spécification soit comme identification.

Ainsi, l'identité $X$ c'est $Y$ se distingue de l'identification et de la spécification sur le plan de la forme par le fait que $\mathrm{X}$ et $\mathrm{Y}$ ont la même forme syntaxique. Cela étant posé, nous proposons des tests permettant de montrer qu'il existe une sous-catégorisation pour l'identité, entre stricte et partielle, alors que l'identification ne peut être que partielle (contra Larreya, 2003). Sur le plan sémantique, on en arrive à la conclusion qu'une phrase copulative à identité est une relation entre $\mathrm{X}$ et $\mathrm{Y}$ posée comme vraie par le locuteur. Notre analyse remet en cause le traitement linguistique en termes de vrai et de faux des phrases copulatives à identité, et permet d'envisager les différents types de phrases copulatives sous un angle nouveau, à travers des principes généraux proposés qui prennent appui sur l'indépendance des notions de référentialité et de prédicativité. Cette piste, à l'état d'ébauche, devrait permettre d'affiner la description de la classe des équatives et de rendre compte du fonctionnement des phrases prédicationnelles et notamment de l'impossibilité d'une phrase comme * Il est un musicien.

\section{Références bibliographiques}

Amary-Coudreau, V. (2010). Qu'est-ce que «c'» est?. Le Français Moderne, vol. 78.2, 259-277.

Amary-Coudreau, V. (2011). S'incarner n'est pas jouer. Description d'un verbe être incarnation. In Le Querler N. et François J. (éd.), Syntaxe et Sémantique, n¹2, 13-48.

Apothéloz, D. (2008). A l'interface du système linguistique et du discours: L'exemple des constructions identificatives (e.g. pseudo-clivées). In Bertrand, O., François, J., Charolles, M., Prévost, S. (éd.), Discours, diachronie, stylistique du français, Etudes en hommage à Bernard Combettes, Berne : Peter Lang, 75-92.

Barbaud, P. (2006). «Prédire n'est pas expliquer» (Thom): la négation de l'infinitif en phrase copulative. In Corblin F., Ferrando S. et Kupferman L. (éd.), Indéfini et Prédication, coll. 'Travaux de Stylistique et de Linguistique françaises: Etudes linguistiques' dirigée par Soutet O., Paris : Presses de l’Université Paris-Sorbonne, 369-388.

Bondarenco, A. (2012). La prédication universelle, prédication subjective et l'ambiguïté dans la phrase simple tautologique et dans la phrase complexe comparative. In Ardeleanu S.-M. (éd.), L'Imaginaire Linguistique et la Dynamique des Langues, ANADISS n ${ }^{\circ} 13: 22-45$.

Charolles, M. \& Schnedecker, C. (1993). Coréférence et identité : le problème des référents évolutifs. In: Langages, 27 e année, $n^{\circ} 112: 106-126$.

Cadiot, P. \& Némo, F. (1997). Pour une sémiogénèse du nom. In Cadiot P. et Habert B. (éd.), Aux sources de la polyphonie nominale, Langue française $113: 24-34$.

Citko, B. (2008). Small Clauses Reconsidered: Not That Small and Not All Alike. Lingua 118 : 261-295.

Culioli, A. \& Desclès, J.-P. (1981). Systèmes de représentations linguistiques et métalinguistiques: les catégories grammaticales et le problème de la description de langues peu étudiées : rapport présenté à l'Unesco, Laboratoire de linguistique formelle, Equipe de recherches associée au CNRS (ERA 642), Université Paris VII, 141 p.

Declerck, R. (1988). Studies on copular sentences, clefts and pseudoclefts, Leuven University, Foris Publications.

Derrida, J. (1971). Le supplément de copule. La philosophie devant la linguistique. Langages, vol. 6, n²4, 14-39.

Desclès, J.-P. \& Froidevaux C. (1982). Axiomatique de la notion de repérage abstrait, Mathématiques et Sciences Humaines, tome $78: 73-119$.

Dikken, M. den (2005). Specificational copular sentences and pseudoclefts. In M. Everaert \& H. van Riemsdijk (éd.), The Blackwell Companion to Syntax. Oxford: Blackwell. Vol. IV, Chapter 6.

Dufaye, L. (2003). Comment identifier une identification ?, L'Identification, Cycnos, Volume $21 \mathrm{n}^{\circ} 1$.

Frege, G. (1994). Ecrits logiques et philosophiques. Ed. Seuil, col. Point Essais.

Gaudin-Bordes, L. (2008). La tyrannie tautologique : l'évidence comme outil énonciatif et stratégie discursive. Langue française, vol. $4 \mathrm{n}^{\circ} 160: 55-7$. 
Gundel, J.K., Hedberg, N. \& Zacharski, R. (1993). Cognitive status and the form of referring expressions in discourse. Language $69: 274-307$

Heggie, L. (1988). The Syntax of Copular Structures. Dissertation, University of Southern California, Los Angeles.

Heycock, C. \& Kroch, A. (1999). Pseudocleft connectivity: Implications for the LF interface level. Linguistic Inquiry 30.3: $365-397$

Heycock, C. \& Kroch, A. (2002). Topic, Focus, and Syntactic Representations. In Mikkelsen, L. \& Potts, C. (éd.), WCCFL 21 Proceedings, Somerville, MA: Cascadilla Press : 141-165.

Higgins, F. R. 1973, The Pseudo-Cleft Construction in English. Thesis, Massachusetts Institute of Technology.

Khalifa, J.-C. (2003). Identification : quelle pertinence pour l'énoncé complexe, L'Identification, Cycnos, Vol. $21 n^{\circ} 1$.

Larreya, P. (2003). Identité et identification, L'Identification, Cycnos, Volume $21 n^{\circ} 1$.

Lee, C. (2002). Contrastive topic and proposition structure. In Sciullo, A.-M., Asymmetry in Grammar, John Benjamins. (Asymmetry Conference, UQAM, 2001) : 345-372.

Le Goffic, P. (1993). Grammaire de la Phrase Française, Paris : Hachette.

Markman, V. G. (2008). Pronomial Copula Constructions Are What? Reduced Specificational Pseudo-Clefts! In Charles B. Chang and Hannah J. Haynie (éd.), Proceedings of the 26th West Coast Conference on Formal Linguistics. Somerville, MA, Cascadilla Proceedings Project : 366-374.

Matushansky, O. \& Spector, B. (2005). Tinker, tailor, soldier, spy. In Maier E., Bary C. \& Huitink, J. (éd.), Proceedings of Sinn und Bedeutung 9: 241-255.

Maurice, L. (2007). La question du rapport entre le sens et la référence dans la philosophie du langage : Le cas des noms propres. Thèse de Philosophie, Université Jean MOULIN - Lyon III.

Mikkelsen, L. (2004). Specifying Who: On the Structure, Meaning, and Use of Specificational Copular Clauses. Ph. D. thesis, University of California, Santa Cruz.

Mikkelsen, L. (2005). Subject choice in copular clauses, ms. UC Berkeley.

Mikkelsen, L. (2011). Copular clauses. In Maienborn C., von Heusinger K., and Portner P. (éd.), Semantics: An International Handbook of Natural Language Meaning, volume 2, Berlin: Mouton de Gruyter, 1805-1829.

Moro, A. (1997). The Raising of Predicates: Predicative Noun Phrases and the Theory of Clause Structure. Cambridge: Cambridge University Press.

Partee, B. H., 1987. Noun phrase interpretation and type-shifting principles. In Groenendijk, J., de Jongh D., \& Stokhof M. (éd.), Studies in Discourse Representation Theory and the Theory of Generalized Quantifiers, vol. 8 of GRASS, Dordrecht: Foris Publications, 115-144.

Paveau M.-A. (2013), Les événements discursifs moraux 4. La linguistique du mensonge de H. Weinrich, La pensée du discours [Carnet de recherche], http://penseedudiscours.hypotheses.org/?p=4777.

Rapoport, T. (1987). Copular, nominal and small clauses. A study of Israeli Hebrew, Diss., MIT.

Reboul, A. (1992). Le paradoxe du mensonge dans la théorie des actes de langage, Cahiers de Linguistique Française $13,125-145$.

Reboul, A. (1997). Combien y a-t-il de poulets ici? Les référents évolutifs, identité et désignation. In Kleiber, G. \& al. (éd.), La continuité référentielle, Paris : Klinsieck, 149-179.

Riegel, M. (2001). Une tautologie est une tautologie, Tautologies logiques et logique des énoncés tautologiques attributifs, Revue d'Etudes Françaises $n^{\circ} 6,135-145$.

Schlenker, P. (2003). Clausal Equations (A Note on the Connectivity Problem), Natural Language and Linguistic Theory 21,157-214.

Van Peteghem, M. (1997). Sur un indéfini marginal : même exprimant l'identité. Langue française. $n^{\circ} 116,61-80$.

Wybraniec-Skardowska, U. (2010). Does the lie contradict the truth? Studies in Logic, Grammar and Rhetoric 20 (33): 1-25. 


\begin{abstract}
* Je remercie les relecteurs anonymes, ainsi qu'Eric Gilbert et Mustapha Krazem, qui ont relu ces pages, pour leurs remarques et commentaires. Ce travail a aussi bénéficié des commentaires de mes collègues du CRISCO. Merci à eux.

${ }^{1}$ Matushansky \& Spector (2005) acceptent la phrase avec et sans le démonstratif. Et on trouve des travaux en philosophie qui traitent de cette phrase sans le démonstratif, par exemple : «Si les noms propres n'avaient pas de sens, alors l'énoncé "Cicéron est Tullius » exprimerait exactement la même chose que l'énoncé "Cicéron est Cicéron ». », cf. Maurice (2007, p. 105).

${ }^{2}$ Pour une revue détaillée de ces différents débats, voir den Dikken (2005) et Mikkelsen (2011).

${ }^{3}$ On utilisera ici XP pour "X-Phrase" ("syntagme X") pour signifier qu'il peut s'agir de n'importe quel type de syntagme.

${ }^{4}$ Selon la terminologie de Higgins (1973), dans une spécificationnelle, le XP1 est superscriptionnel et le XP2 est spécificationnel, alors que dans une identification, le XP1 est référentiel et le XP2 est identificationnel. Mais les définitions des termes superscriptionnel, spécificationnel et identificationnel restent floues malgré les descriptions très fines de Higgins (1973). Le lien entre les notions qu'il propose et la référentialité n'est pas très précis.
\end{abstract}

${ }^{5}$ En effet, en 2006, on a considéré que Pluton ne correspondait pas à la définition d'une planète, et le nombre de planètes recensées est passé de neuf à huit.

${ }^{6}$ Cette phrase peut être améliorée par l'insertion de $d e$, bien que ce type de phrase ne nous semble pas très naturel ( ? Le nombre de planètes est de neuf.). En tant que phrase spécificationnelle (cf. Higgins, 1973), cette phrase devrait pouvoir apparaître avec le démonstratif $c^{\prime}$, ce qui semble être le cas bien que la phrase ainsi formée nous semble encore un peu bizarre: ?Le nombre de planète, c'est neuf. Les phrases avec des nombres, en français, semblent avoir un comportement assez particulier, que nous ne développerons pas ici.

${ }^{7}$ Cela ne veut pas dire que les autres « animaux » ne mentent pas, d'une façon ou d'une autre, mais nous laissons ce point de côté.

${ }^{8}$ Il existe quelques études sur le mensonge, cf. Wybraniec-Skardowska (2010), Reboul (1992) et voir aussi un article de Paveau (2013) sur le site http://penseedudiscours.hypotheses.org/?p=4777. Néanmoins le mensonge n'est pas un thème de recherche très courant en linguistique.

${ }^{9}$ Mais il existe des contextes où un nom propre peut être un prédicat : les contextes de films, par exemple.

${ }^{10}$ On laissera de côté ici des éléments comme Ce que $P$, ça ou encore les infinitifs.

${ }^{11}$ Heggie (1988) propose une hiérarchie pour évaluer ce qui peut servir d'attribut à chaque type XP en fonction de leurs natures référentielles respectives. Ce continuum diffère de la notion de Givenness Hierarchy de Gundel \& al. (1993).

${ }^{12}$ Ceci n'est qu'une ébauche pour le français, et nous pensons qu'il y a des variations en fonction des langues.

${ }^{13}$ On peut leur appliquer les tests précédents, les résultats sont ceux de l'identité stricte :

(i) a. \#Un loup c'est un poisson, mais c'est aussi un bar. b. \#Le loup c'est un poisson, mais c'est aussi le bar. (ii) a. \#Un loup c'est un bar, mais ça n'est pas que ça. $\quad$ b. \#Le loup c'est le bar, mais ça n'est pas que ça.

${ }^{14}$ Cf. Frege, 1994 : 105 : «La représentation associée à un signe doit être distinguée de la dénotation et du sens de ce signe. Si un signe dénote un objet perceptible au moyen des sens, ma représentation est un tableau intérieur, formé du souvenir des impressions sensibles et des actions externes ou internes auxquelles je me suis livré. Dans ce tableau, les sentiments pénètrent les représentations ; la distinction de ces diverses partie est inégale et inconstante. Chez le même individu, la même représentation n'est pas toujours liée au même sens. Car la représentation est subjective ; celle de l'un n'est pas celle de l'autre. Et il est bien naturel que les représentations associées au même sens diffèrent grandement entre elles. » 